\title{
The Pipeline From Abstract Presentation to Publication in Pediatric Hospital Medicine
}

\author{
Lisa E. Herrmann, MD, MEd ${ }^{1 *}$, Matthew Hall, PhD², Kathryn Kyler, MD³, Joseph Cochran², Annie L. Andrews, MD, MSCR4, \\ Derek J. Williams, MD, MPH${ }^{5}$, Karen M. Wilson, MD, MPH'ㄴ, Samir S. Shah, MD, MSCE${ }^{1}$, \\ for the Pediatric Research in Inpatient Settings (PRIS) Network
}

\begin{abstract}
${ }^{1}$ Division of Hospital Medicine, Cincinnati Children's Hospital Medical Center, Cincinnati, Ohio; ${ }^{2}$ Children's Hospital Association, Lenexa, Kansas; ${ }^{3}$ Department of Pediatrics, Children's Mercy Hospital, Kansas City, Missouri; ${ }^{4}$ Department of Pediatrics, Medical University of South Carolina, Charleston, South Carolina; ${ }^{5}$ Department of Pediatrics, Vanderbilt University School of Medicine and Vanderbilt University Medical Center, Nashville, Tennessee; ${ }^{6}$ Department of Pediatrics, Icahn School of Medicine at Mount Sinai, New York, New York.
\end{abstract}

BACKGROUND: The annual Pediatric Hospital Medicine (PHM) conference serves as a venue for the dissemination of research in this rapidly growing discipline. A measure of research validity is subsequent publication in peer-reviewed journals.

OBJECTIVE: To identify the publication rate of abstracts submitted to the 2014 PHM conference and determine whether presentation format was associated with subsequent journal publication or time to publication.

METHODS: We identified abstracts submitted to the 2014 PHM conference. Presentation formats included rejected abstracts and poster and oral presentations. Abstracts subsequently published in journals were identified by searching the author and abstract title in PubMed, MedEdPORTAL, and Google Scholar. We used logistic regression and Cox proportional hazards models to determine if presentation format was associated with publication, time to publication, and publishing journal impact factor.
RESULTS: Of 226 submitted abstracts, $19.0 \%$ were rejected, $68.0 \%$ were selected for posters, and $12.8 \%$ were selected for oral presentations; $36.3 \%$ were subsequently published within 30 months after the conference. Abstracts accepted for oral presentation had more than 7-fold greater odds of publication (adjusted odds ratio 7.8; $95 \%$ confidence interval [Cl], 2.6-23.5) and a 4-fold greater likelihood of publication at each month (adjusted hazard ratio $4.5 ; 95 \% \mathrm{Cl}, 2.1-9.7)$ compared with rejected abstracts. Median journal impact factor was significantly higher for oral presentations than other presentation formats $(P<.01)$.

CONCLUSIONS: Abstract reviewers may be able to identify methodologically sound studies for presentation; however, the low overall publication rate may indicate that presented results are preliminary or signify a need for increased mentorship and resources for research development in PHM. Journal of Hospital Medicine 2018;13:90-95. Published online first October 3, 2017. (C) 2018 Society of Hospital Medicine ediatric hospital medicine (PHM) is one of the most rapidly growing disciplines in pediatrics, ${ }^{1}$ with $8 \%$ of pediatric residency graduates each year entering the field. ${ }^{2}$ Research plays an important role in advancing care in the field and is a critical component for board certification and fellowship accreditation. ${ }^{3-6}$ The annual PHM conference, which has been jointly sponsored by the Academic Pediatric Association, the American Academy of Pediatrics, and the Society of Hospital Medicine, is an important venue for the dissemination of research findings. Abstract selection is determined by peer review; however, reviewers are provided

\footnotetext{
*Address for correspondence and reprint requests: Lisa E. Herrmann, MD, MEd, Division of Hospital Medicine, Cincinnati Children's Hospital Medical Center, 3333 Burnet Avenue, MLC 9016, Cincinnati, OH 45229; Telephone: 513803-4257; Fax: 513-803-9244; E-mail: lisa.herrmann@cchmc.org

Received: February 9, 2017; Revised: June 9, 2017;

Accepted: June 23, 2017
}

2018 Society of Hospital Medicine DOI 10.12788/jhm.2853 with only a brief snapshot of the research, which may not contain sufficient information to fully evaluate the methodological quality of the work. ${ }^{7-10}$ Additionally, while instructions are provided, reviewers often lack formal training in abstract review. Consequently, scores may vary. ${ }^{9}$

Publication in a peer-reviewed journal is considered a measure of research success because it requires more rigorous peer review than the abstract selection process at scientific meetings. ${ }^{11-16}$ Rates of subsequent journal publication differ based on specialty and meeting, and they have been reported at $23 \%$ to $78 \%{ }^{10,12,14-18}$ In pediatrics, publication rates after presentation at scientific meetings range from $36 \%$ to $63 \%$, with mean time to publication ranging from 20 to 26 months following the meeting. ${ }^{11,19,20}$ No studies have reviewed abstract submissions to the annual PHM meeting to determine if selection or presentation format is associated with subsequent publication in a peer-reviewed journal.

We sought to identify the publication rate of abstracts submitted to the 2014 PHM conference and determine whether presentation format was associated with the likelihood of subsequent journal publication or time to publication. 


\section{METHODS}

\section{Study Design}

Data for this retrospective cohort study were obtained from a database of all abstracts submitted for presentation at the 2014 PHM conference in Lake Buena Vista, Florida.

\section{Main Exposures}

The main exposure was presentation format, which was categorized as not presented (ie, rejected), poster presentation, or oral presentation. PHM has a blinded abstract peer-review process; in 2014, an average of 10 reviewers scored each abstract. Reviewers graded abstracts on a scale of 1 (best in category) to 7 (unacceptable for presentation) according to the following criteria: originality, scientific importance, methodological rigor, and quality of presentation. Abstracts with the lowest average scores in each content area, usually less than or equal to 3, were accepted as oral presentations while most abstracts with scores greater than 5 were rejected. For this study, information collected from each abstract included authors, if the primary author was a trainee, title, content area, and presentation format. Content areas included clinical research, educational research, health services research (HSR) and/or epidemiology, practice management research, and quality improvement. Abstracts were then grouped by presentation format and content area for analysis. The Pediatric Academic Societies (PAS) annual meeting, another common venue for the presentation of pediatric research, precedes the PHM conference. Because acceptance for PAS presentation may represent more strongly developed abstract submissions for PHM, we identified which abstracts had also been presented at the PAS conference that same year by cross-referencing authors and abstract titles with the PAS 2014 program.

\section{Main Outcome Measures}

All submissions were assessed for subsequent publication in peer-reviewed journals through January 2017 (30 months following the July 2014 PHM conference). To identify abstracts that went on to full publication, 2 authors (JC and LEH) independently searched for the lead author's name and the presentation title in PubMed, Google Scholar, and MedEdPORTAL in January 2017. PubMed was searched using both the general search box and an advanced search for author and title. Google Scholar was added to capture manuscripts that may not have been indexed in PubMed at the time of our search. MedEdPORTAL, a common venue for the publication of educational initiatives that are not currently indexed in PubMed, was searched by lead author name via the general search box. If a full manuscript was published discussing similar outcomes or results and was written by the same authors who had submitted a PHM conference abstract, it was considered to have been published. The journal, month, and year of publication were recorded. For journals published every 2 months, the date of publication was recorded as falling between the 2 months. For those journals with biannual publication in the spring and fall, the months of March and October were used, respectively. The impact factor of the publication journal was also recorded for the year preceding publication. A journal's impact factor is frequently used as a quantitative measure of journal quality and reflects the frequency with which a journal's articles are cited in the scientific literature. ${ }^{21}$ Journals without an impact factor (eg, newer journals) were assigned a 0.

\section{Data Analysis}

All abstracts submitted to the PHM conference were analyzed based on content area and presentation format. The proportion of all abstracts subsequently published was determined for each format type and content area, and the odds ratio (OR) for publication after abstract submission was calculated using logistic regression. We calculated an adjusted OR for subsequent publication controlling for PAS presentation and the trainee status of the primary author. The journals most frequently publishing abstracts submitted to the PHM conference were identified. Median time to publication was calculated using the number of months elapsed between the PHM conference and publication date and compared across all abstract formats using Cox proportional hazards models adjusted for PAS presentation and trainee status. Kaplan-Meier survival curves were also generated for each of the 3 formats and compared using log-rank tests. The median impact factor was determined for each abstract format and compared using Wilcoxon rank-sum tests. Median impact factor by content area was compared using a Kruskal-Wallis test. All statistical analyses were performed using SAS version 9.2 (SAS Institute, Cary, NC). $P$ values $<.05$ were considered statistically significant. In accordance with the Common Rule 22 and the policies of the Cincinnati Children's Hospital Medical Center Institutional Review Board, this research was not considered human subjects research.

\section{RESULTS}

For the 2014 PHM meeting, 226 abstracts were submitted, of which 183 (81.0\%) were selected for presentation, including 154 (68.0\%) as poster presentations and 29 (12.8\%) as oral presentations. Of all submitted abstracts, 82 (36.3\%) were published within 30 months following the meeting. Eightyone of these (98.8\%) were identified via PubMed, and 1 was found only in MedEdPORTAL. No additional publications were found via Google Scholar. The presenting author for the PHM abstract was the first author for $87.8 \%(n=72)$ of the publications. A trainee was the presenting author for only 2 of these abstracts. For the publications in which the first author was not the presenting author, the presenting author was the senior author in 2 of the publications and the second or third author on the remaining 8 . Of the abstracts accepted for presentation, 70 (38.3\%) were subsequently published. Abstracts accepted for oral presentation had almost 7-fold greater odds of subsequent publication than those that were rejected (Table 1; OR 6.8; 95\% confidence interval [Cl], 2.4-19.4). Differences in the odds of publication for rejected abstracts compared with those accepted for poster presentation were not statistically significant (OR 1.2; 95\% Cl, 0.5-2.5).

Of the abstracts submitted to PHM, 118 (52.2\%) were also presented at the 2014 PAS meeting. Of these, 19 (16.1\%) were 
TABLE 1. Publication Rates by Presentation Type and Content Area

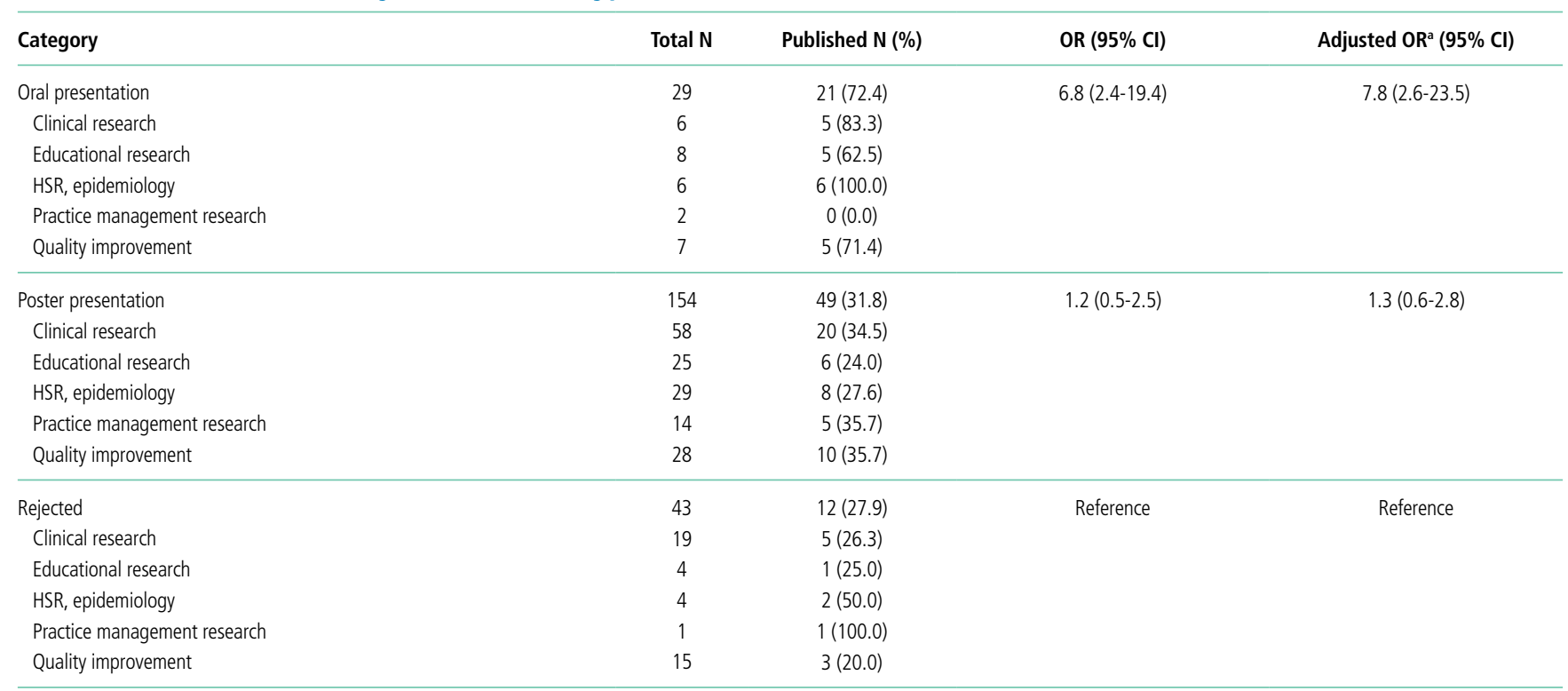

adjusted for presentation at the Pediatric Academic Societies conference and a trainee as the first author. NOTE: Abbreviations: $\mathrm{Cl}$, confidence interval; HSR, health services research; OR, odds ratio.

TABLE 2. Months to Publication and Impact Factor of Journal in Which Publication Appeared by Abstract Presentation Type

\begin{tabular}{|c|c|c|c|c|c|c|}
\hline Presentation Type & $\begin{array}{l}\text { Months to Publication } \\
\text { Median (IQR) }\end{array}$ & $P$ & $\begin{array}{l}\text { Unadjusted HR } \\
(95 \% \mathrm{Cl})\end{array}$ & $\begin{array}{l}\text { Adjusted } \text { HR }^{\mathrm{a}} \\
(95 \% \mathrm{Cl})\end{array}$ & Impact Factor Median (IQR) & $P$ \\
\hline Oral & $15(9-18)$ & .431 & $3.8(1.8-7.8)$ & $4.5(2.1-9.7)$ & $5.3(2.0-5.3)$ & .008 \\
\hline Poster & $20(15-22)$ & .029 & $1.1(0.5-2.0)$ & $1.1(0.6-2.2)$ & $0.3(0.0-1.8)$ & .339 \\
\hline Rejected & $9.5(3-17.5)$ & Reference & Reference & Reference & $1.2(0.4-1.5)$ & Referenc \\
\hline
\end{tabular}

${ }^{a}$ Adjusted for presentation at the Pediatric Academic Societies conference and a trainee as the first author. NOTE: Abbreviations: $\mathrm{Cl}$, confidence interval; $\mathrm{HR}$, hazard ratio; $I \mathrm{QR}$, interquartile range.

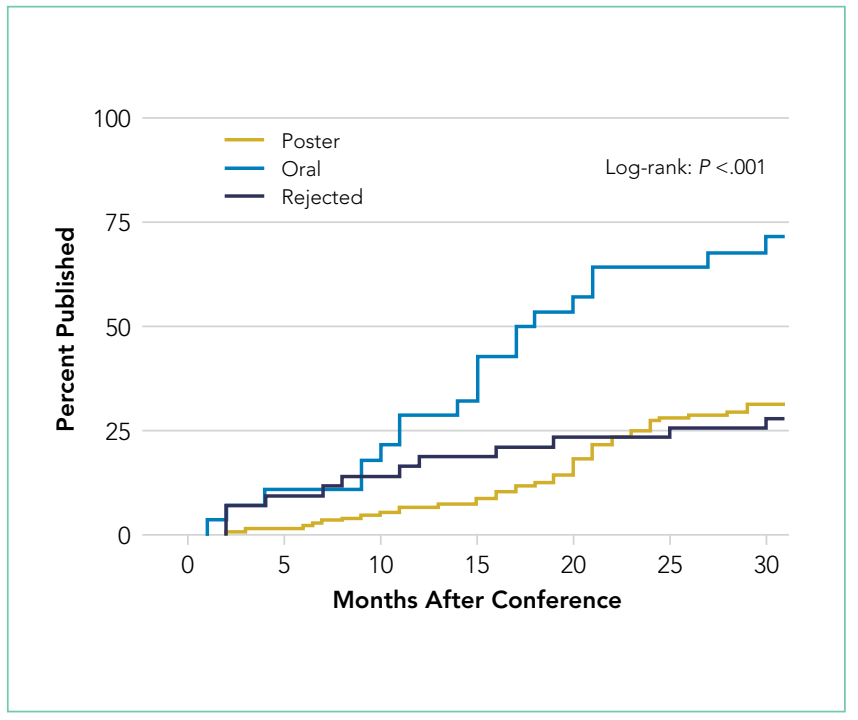

FIG. Cumulative frequency distribution for months to publication by type of abstract presentation. rejected from PHM, 79 (66.9\%) were accepted for poster presentation, and 20 (16.9\%) were accepted for oral presentation. A trainee was the primary author for $40.3 \%(n=91)$ of the abstracts submitted to PHM; abstracts submitted by trainees were more likely to be rejected from conference presentation $(P=.002)$. Of the abstracts submitted by a trainee, 7 (24.1\%) were accepted for oral presentation, 57 (37.0\%) were accepted for poster presentation, and 27 (63\%) were rejected from presentation. Adjusting for presentation at PAS and trainee status did not substantively change the odds of subsequent publication for abstracts accepted for poster presentation, but it increased the odds of publication for abstracts accepted for oral presentation (Table 1).

Of the abstracts subsequently published in journals, the median time to publication was 17 months (interquartile range [IQR], 10-21; Table 2, Figure). Abstracts accepted for oral presentation had an almost 4-fold greater likelihood of publication at each month than rejected abstracts (Table 2). Among abstracts that were subsequently published, the median jour- 
nal impact factor was significantly higher for abstracts accepted for oral presentation than for either rejected abstracts or those accepted for poster presentation (Table 2). The median impact factor by content area was as follows: clinical research 1.0, educational research 2.1, HSR and epidemiology 1.5, practice management research 0 , and quality improvement 1.4 ( $P$ $=0.023)$. The most common journals were Hospital Pediatrics (31.7\%, $n=26)$, Pediatrics (15.9\%, $n=13$ ), and the Journal of Hospital Medicine $(4.9 \%, n=4)$. Oral presentation abstracts were most commonly published in Pediatrics, Hospital Pediatrics, and JAMA Pediatrics. Hospital Pediatrics was the most common journal for abstracts accepted for poster presentation, representing $44.9 \%$ of the published abstracts. Rejected abstracts were subsequently published in a range of journals, including Clinical Pediatrics, Advances in Preventative Medicine, and Ethnicity \& Disease (Table 3).

\section{DISCUSSION}

About one-third of abstracts submitted to the 2014 PHM conference were subsequently published in peer-reviewed journals within 30 months of the conference. Compared with rejected abstracts, the rate of publication was significantly higher for abstracts selected for oral presentation but not for those selected for poster presentation. For abstracts ultimately published in journals, selection for oral presentation was significantly associated with both a shorter time to publication and a higher median journal impact factor compared with rejected abstracts. Time to publication and median journal impact factor were similar between rejected abstracts and those accepted for poster presentation. Our findings suggest that abstract reviewers may be able to identify which abstracts will ultimately withstand more stringent peer review in the publication process when accepting abstracts for oral presentation. However, the selection for poster presentation versus rejection may not be indicative of future publication or the impact factor of the subsequent publication journal.

Previous studies have reviewed publication rates after meetings of the European Society for Pediatric Urology (publication rate of 47\%), ${ }^{11}$ the Ambulatory Pediatric Association (now the Academic Pediatric Association; publication rate of $47 \%$ ), the American Pediatric Society/Society for Pediatric Research (publication rate of 54\%), and the PAS (publication rate of $45 \%) .{ }^{19,20}$ Our lower publication rate of $36.3 \%$ may be attributed to the shorter follow-up time in our study (30 months from the PHM conference), whereas prior studies monitored for publication up to 60 months after the PAS conference. ${ }^{20}$ Factors associated with subsequent publication include statistically significant results, a large sample size, and a randomized controlled trial study design. ${ }^{15,16}$ The primary reason for nonpublication for up to $80 \%$ of abstracts is failure to submit a manuscript for publication. ${ }^{23}$ A lack of time and fear of rejection after peer review are commonly cited explanations. ${ }^{18,23,24}$ Individuals may view acceptance for an oral presentation as positive reinforcement and be more motivated to pursue subsequent manuscript publication than individuals whose abstracts are offered poster presentations or are
TABLE 3. Journals in Which Manuscripts Were Published by Abstract Presentation Type

\begin{tabular}{|c|c|c|}
\hline Presentation Type & Journal & $\mathrm{N}(\%)$ \\
\hline \multirow[t]{4}{*}{ Oral } & Pediatrics & $10(47.6)$ \\
\hline & Hospital Pediatrics & $4(19.1)$ \\
\hline & JAMA Pediatrics & $2(9.5)$ \\
\hline & Other & $5(23.8)$ \\
\hline \multirow[t]{5}{*}{ Poster } & Hospital Pediatrics & $22(44.9)$ \\
\hline & Journal of Hospital Medicine & $3(6.1)$ \\
\hline & Pediatrics & $2(4.1)$ \\
\hline & Pediatric Emergency Care & $2(4.1)$ \\
\hline & Other & $20(40.8)$ \\
\hline \multirow[t]{11}{*}{ Rejected } & Clinical Pediatrics & $2(16.7)$ \\
\hline & Advances in Preventive Medicine & $1(8.3)$ \\
\hline & Ethnicity \& Disease & $1(8.3)$ \\
\hline & Frontiers in Pediatrics & $1(8.3)$ \\
\hline & Indian Journal of Pediatrics & $1(8.3)$ \\
\hline & Journal of Clinical Research in Pediatric Endocrinology & $1(8.3)$ \\
\hline & Journal of Hypertension & $1(8.3)$ \\
\hline & Journal of Tropical Pediatrics & $1(8.3)$ \\
\hline & Pain Management Nursing & $1(8.3)$ \\
\hline & Pediatrics & $1(8.3)$ \\
\hline & Pediatrics and Neonatology & $1(8.3)$ \\
\hline
\end{tabular}

Abbreviation: JAMA, Journal of the American Medical Association.

rejected. Trainees frequently present abstracts at scientific meetings, representing $40.3 \%$ of primary authors submitting abstracts to PHM in 2014, but may not have sufficient time or mentorship to develop a complete manuscript. ${ }^{18}$ To our knowledge, there have been no publications that assess the impact of trainee status on subsequent publication after conference submission.

Our study demonstrated that selection for oral presentation was associated with subsequent publication, shorter time to publication, and publication in journals with higher impact factors. A 2005 Cochrane review also demonstrated that selection for oral presentation was associated with subsequent journal publication. ${ }^{16}$ Abstracts accepted for oral publication may represent work further along in the research process, with more developed methodology and results. The shorter time to publication for abstracts accepted for oral presentation could also reflect feedback provided by conference attendees after the presentation, whereas poster sessions frequently lack a formalized process for critique.

Carroll et al. found no difference in time to publication be- 
tween abstracts accepted for presentation at the PAS and rejected abstracts. ${ }^{20}$ Previous studies demonstrate that most abstracts presented at scientific meetings that are subsequently accepted for publication are published within 2 to 3 years of the meeting ${ }^{12}$ with publication rates as high as $98 \%$ within 3 years of presentation. ${ }^{17}$ In contrast to Carroll et al., we found that abstracts accepted for oral presentation had a 4-fold greater likelihood of publication at each month than rejected abstracts. However, abstracts accepted for poster presentation did not have a significant difference in the proportional hazard ratio models for publication compared with rejected abstracts. Because space considerations limit the number of abstracts that can be accepted for presentation at a conference, some abstracts that are suitable for future publication may have been rejected due to a lack of space. Because researchers often use scientific meetings as a forum to receive peer feedback, ${ }^{12}$ authors who present at conferences may take more time to write a manuscript in order to incorporate this feedback.

The most common journal in which submitted abstracts were subsequently published was Hospital Pediatrics, representing twice as many published manuscripts as the second most frequent journal, Pediatrics. Hospital Pediatrics, which was first published in 2011, did not have an impact factor assigned during the study period. Yet, as a peer-reviewed journal dedicated to the field of PHM, it is well aligned with the research presented at the PHM meeting. It is unclear if Hospital Pediatrics is a journal to which pediatric hospitalists tend to submit manuscripts initially or if manuscripts are frequently submitted elsewhere prior to their publication in Hospital Pediatrics. Submission to other journals first likely extends the time to publication, especially for abstracts accepted for poster presentation, which may describe studies with less developed methods or results.

This study has several limitations. Previous studies have demonstrated mean time to publication of 12 to 32 months following abstract presentation with a median time of 19.6 months. ${ }^{16}$ Because we only have a 30 -month follow-up, there may be abstracts still in the review process that are yet to be published, especially because the length of the review process varies by journal. We based our literature search on the first author of each PHM conference abstract submission, assuming that this presenting author would be one of the publishing authors even if not remaining first author; if this was not the case, we may have missed some abstracts that were subsequently published in full. Likewise, if a presenting author's last name changed prior to the publication of a manuscript, a publication may have been missed. This limitation would cause us to underestimate the overall publication rate. It is not clear whether this would differentially affect the method of presentation. However, in this study, there was concordance between the presenting author and the publication's first author in $87.8 \%$ of the abstracts subsequently published in full. Presenting authors who did not remain the first author on the published manuscript maintained authorship as either the senior author or second or third author, which may represent changes in the degree of involvement or a division of responsibilities for indi- viduals working on a project together. While our search methods were comprehensive, there is a possibility that abstracts may have been published in a venue that was not searched. Additionally, we only reviewed abstracts submitted to PHM for 1 year. As the field matures and the number of fellowship programs increases, the quality of submitted abstracts may increase, leading to higher publication rates or shorter times to publication. It is also possible that the publication rate may not be reflective of PHM as a field because hospitalists may submit their work to conferences other than the PHM. Lastly, it may be more challenging to interpret any differences in impact factor because some journals, including Hospital Pediatrics (which represented a plurality of poster presentation abstracts that were subsequently published and is a relatively new journal), did not have an impact factor assigned during the study period. Assigning a 0 to journals without an impact factor may artificially lower the average impact factor reported. Furthermore, an impact factor, which is based on the frequency with which an individual journal's articles are cited in scientific or medical publications, may not necessarily reflect a journal's quality.

\section{CONCLUSIONS}

Of the 226 abstracts submitted to the 2014 PHM conference, approximately one-third were published in peer-reviewed journals within 30 months of the conference. Selection for oral presentation was found to be associated with subsequent publication as well as publication in journals with higher impact factors. The overall low publication rate may indicate a need for increased mentorship and resources for research development in this growing specialty. Improved mechanisms for author feedback at poster sessions may provide constructive suggestions for further development of these projects into full manuscripts or opportunities for trainees and early-career hospitalists to network with more experienced researchers in the field.

Disclosure: Drs. Herrmann, Hall, Kyler, Andrews, Williams, and Shah and Mr. Cochran have nothing to disclose. Dr. Wilson reports personal fees from the American Academy of Pediatrics during the conduct of the study. The authors have no financial relationships relevant to this article to disclose.

\section{References}

1. Stucky ER, Ottolini MC, Maniscalco J. Pediatric hospital medicine core competencies: development and methodology. J Hosp Med. 2010;5(6):339-343.

2. Freed GL, McGuinness GA, Althouse LA, Moran LM, Spera L. Long-term plans for those selecting hospital medicine as an initial career choice. Hosp Pediatr. 2015;5(4):169-174.

3. Rauch D. Pediatric Hospital Medicine Subspecialty. 2016; https://www.aap. org/en-us/about-the-aap/Committees-Councils-Sections/Section-on-Hospital-Medicine/Pages/Pediatric-Hospital-Medicine-Subspecialty.aspx. Accessed November 28, 2016.

4. Bekmezian A, Teufel RJ, Wilson KM. Research needs of pediatric hospitalists. Hosp Pediatr. 2011;1(1):38-44.

5. Teufel RJ, Bekmezian A, Wilson K. Pediatric hospitalist research productivity: predictors of success at presenting abstracts and publishing peer-reviewed manuscripts among pediatric hospitalists. Hosp Pediatr. 2012;2(3):149-160.

6. Wilson KM, Shah SS, Simon TD, Srivastava R, Tieder JS. The challenge of pediatric hospital medicine research. Hosp Pediatr. 2012;2(1):8-9.

7. Froom P, Froom J. Presentation Deficiencies in structured medical abstracts. J Clin Epidemiol. 1993;46(7):591-594 
8. Relman AS. News reports of medical meetings: how reliable are abstracts? N Engl J Med. 1980;303(5):277-278.

9. Soffer A. Beware the 200-word abstract! Arch Intern Med. 1976;136(11): 1232-1233.

10. Bhandari M, Devereaux P, Guyatt GH, et al. An observational study of orthopaedic abstracts and subsequent full-text publications. J Bone Joint Surg Am. 2002;84(4):615-621.

11. Castagnetti M, Subramaniam R, El-Ghoneimi A. Abstracts presented at the European Society for Pediatric Urology (ESPU) meetings (2003-2010): Characteristics and outcome. J Pediatr Urol. 2014;10(2):355-360.

12. Halikman R, Scolnik D, Rimon A, Glatstein MM. Peer-Reviewed Journa Publication of Abstracts Presented at an International Emergency Medicine Scientific Meeting: Outcomes and Comparison With the Previous Meeting. Pediatr Emerg Care. 2016.

13. Relman AS. Peer review in scientific journals--what good is it? West J Med. 1990;153(5):520.

14. Riordan F. Do presenters to paediatric meetings get their work published? Arch Dis Child. 2000;83(6):524-526.

15. Scherer RW, Dickersin K, Langenberg P. Full publication of results initially presented in abstracts: a meta-analysis. JAMA. 1994;272(2):158-162.

16. Scherer RW, Langenberg P, Elm E. Full publication of results initially presented in abstracts. Cochrane Database Syst Rev. 2005.

17. Marx WF, Cloft HJ, Do HM, Kallmes DF. The fate of neuroradiologic abstracts presented at national meetings in 1993: rate of subsequent publication in peer-reviewed, indexed journals. Am J Neuroradiol. 1999;20(6):1173-1177.

18. Roy D, Sankar V, Hughes J, Jones A, Fenton J. Publication rates of scientific papers presented at the Otorhinolarygological Research Society meetings. Clin Otolaryngol Allied Sci. 2001;26(3):253-256.

19. McCormick MC, Holmes JH. Publication of research presented the pediatric meetings: change in selection. Am J Dis Child. 1985;139(2):122-126.

20. Carroll AE, Sox CM, Tarini BA, Ringold S, Christakis DA. Does presentation format at the Pediatric Academic Societies' annual meeting predict subsequent publication? Pediatrics. 2003;112(6):1238-1241.

21. Saha S, Saint S, Christakis DA. Impact factor: a valid measure of journal quality? J Med Libr Assoc. 2003;91(1):42.

22. Office for Human Research Protections. Code of Federal Regulations, Title 45 Public Welfare: Part 46, Protection of Human Subjects, $\$ 46.102(\mathrm{f})$. http:// www.hhs.gov/ohrp/regulations-and-policy/regulations/45-cfr-46/index. html\#46.102. Accessed October 21, 2016.

23. Weber EJ, Callaham ML, Wears RL, Barton C, Young G. Unpublished research from a medical specialty meeting: why investigators fail to publish. JAMA. 1998;280(3):257-259.

24. Timmer A, Hilsden RJ, Cole J, Hailey D, Sutherland LR. Publication bias in gastroenterological research-a retrospective cohort study based on abstracts submitted to a scientific meeting. BMC Med Res Methodol. 2002;2(1):1. 